\title{
Viabilidade econômica da geração de energia elétrica em motor dual biogás/diesel no meio rural
}

\author{
Economic feasibility of electric power generation in a dual biogas-diesel engine in rural areas \\ Viabilidad económica de la generación de energía eléctrica en un motor dual biogás-diesel en zonas
}

rurales

Recebido: 08/12/2021 | Revisado: 12/12/2021 | Aceito: 14/12/2021 | Publicado: 22/12/2021

\author{
Juliano de Souza \\ ORCID: https://orcid.org/0000-0002-1992-8410 \\ Universidade Estadual do Oeste do Paraná, Brasil \\ E-mail: melegsouza@gmail.com \\ Samuel Nelson Melegari de Souza \\ ORCID: https://orcid.org/0000-0002-3581-902X \\ Universidade Estadual do Oeste do Paraná, Brasil \\ E-mail: samuel.souza@unioeste.br \\ Doglas Bassegio \\ ORCID: https://orcid.org/0000-0001-6628-8594 \\ Universidade Estadual do Oeste do Paraná, Brasil \\ E-mail: doglas.bassegio@unioeste.br \\ Deonir Secco \\ ORCID: https://orcid.org/0000-0002-3042-159X \\ Universidade Estadual do Oeste do Paraná, Brasil \\ E-mail: deonir.secco@unioeste.br \\ Victor Vaz \\ ORCID: https://orcid.org/0000-0001-5285-1286 \\ Universidade Estadual do Oeste do Paraná, Brasil \\ E-mail: victorvaz.fis@gmail.com \\ Willian Cézar Nadaleti \\ ORCID: https://orcid.org/0000-0002-4727-4127 \\ Universidade Federal de Pelotas, Brasil \\ E-mail: williancezarnadaletti@ gmail.com \\ Flávio Gurgacz \\ ORCID: https://orcid.org/0000-0002-4001-7072 \\ Universidade Estadual do Oeste do Paraná, Brasil \\ E-mail: flavio.gurgcz@unioeste.br \\ Waldir Mariano Machado Junior \\ ORCID: https://orcid.org/0000-0001-6722-6600 \\ Universidade Estadual do Oeste do Paraná, Brasil \\ E-mail: waldir.junior5@unioeste.br
}

\begin{abstract}
Resumo
O biogás resultante da digestão anaeróbia de resíduos agrícolas é interessante para geração própria de eletricidade. Com a normatização de micro e mini geração distribuída de eletricidade em 2012 no Brasil, abriu-se uma grande oportunidade para uso de biomassa e energia solar para geração de eletricidade renovável em propriedades rurais. O estudo de viabilidade das tecnologias de uso do biogás na geração de eletricidade é importante em tomadas de decisões. Geralmente a geração de eletricidade com biogás é realizada em motores geradores a gás com ignição por compressão. Neste estudo é proposto a utilização de motores dual ciclo diesel, o qual utiliza simultaneamente dois combustíveis, gás e diesel ou biodiesel. Este trabalho teve como objetivo geral a análise da viabilidade econômica da utilização do motor dual biogás/diesel, na produção de energia elétrica para meio rural. Foram analisadas três condições: motor gerador com $100 \%$ de diesel, $50 \%$ diesel e $50 \%$ biogás, $90 \%$ biogás e $10 \%$ diesel, operando 5 , 10 ou 20 horas por dia. Os resultados gerados comprovaram a viabilidade dessa prática somente com motores geradores de potência $50 \mathrm{~kW}$ ou mais operando no mínimo 10 horas por dia e ajustado para consumir $90 \%$ de biogás e $10 \%$ de diesel. Observou-se que os custos de geração dependem da economia de escala.
\end{abstract}

Palavras-chave: Energia; Biomassa; Eletricidade.

\section{Abstract}

The biogas resulting from the anaerobic digestion of agricultural residues is interesting for its own generation of electricity. With the standardization of micro and mini distributed electricity generation in 2012 at Brazil, a great opportunity was opened for the use of biomass and solar energy to generate renewable electricity in rural areas. The 
feasibility study of technologies for using biogas in electricity generation is important in decision-making. Generally the generation of electricity with biogas is carried out in gas generator engines with compression ignition. In this study, the use of dual-cycle diesel engines is proposed, which simultaneously use two fuels, gas and diesel or biodiesel. This work had as general objective the analysis of the economic viability of using the dual biogas/diesel engine in the production of electric energy for rural areas. Three conditions were analyzed: generator engine with $100 \%$ diesel, $50 \%$ diesel and 50\% biogas, $90 \%$ biogas and 10\% diesel, operating 5, 10 or 20 hours a day. The results proved the feasibility of this practice only with generator engines with a power of $50 \mathrm{~kW}$ or more, operating at least 10 hours a day and adjusted to consume $90 \%$ biogas and $10 \%$ diesel. It was observed that generation costs depend on economies of scale.

Keywords: Energy; Biomass; Electricity.

\section{Resumen}

El biogás resultante de la digestión anaeróbica de residuos agrícolas es interesante para su propia generación de electricidad. Con la estandarización de la generación de electricidad micro y mini distribuida en 2012 en Brasil, se abrió una gran oportunidad para el uso de biomasa y energía solar para generar electricidad renovable en propiedades rurales. El estudio de viabilidad de tecnologías para el uso de biogás en la generación de electricidad es importante en la toma de decisiones. Generalmente la generación de electricidad con biogás se realiza en motores generadores de gas con encendido por compresión. En este estudio se propone el uso de motores diesel de ciclo dual, que utilizan simultáneamente dos combustibles, gas y diesel o biodiesel. Este trabajo tuvo como objetivo general el análisis de la viabilidad económica del uso del motor dual biogás / diesel en la producción de energía eléctrica para áreas rurales. Se analizaron tres condiciones: motor generador con $100 \%$ diésel, $50 \%$ diésel y $50 \%$ biogás, $90 \%$ biogás y $10 \%$ diésel, operando 5, 10 o 20 horas diarias. Los resultados generados demostraron la viabilidad de esta práctica solo con motores generadores con una potencia de $50 \mathrm{~kW}$ o más operando al menos 10 horas al día y ajustados para consumir 90\% de biogás y 10\% de diesel. Se observó que los costos de generación dependen de las economías de escala.

Palabras clave: Energía; Biomasa; Electricidad.

\section{Introdução}

Devido principalmente ao uso de combustíveis fósseis (carvão, petróleo e gás natural) em atividades humanas, as emissões de dióxido de carbono $\left(\mathrm{CO}_{2}\right)$ vem aumentando gradativamente há anos. A decomposição de matéria orgânica, pecuária e outras atividades agrícolas vem contribuindo para as emissões de metano $\left(\mathrm{CH}_{4}\right)$. $\mathrm{O} \mathrm{CO}_{2}$ e $\mathrm{CH}_{4}$ são gases que vem contribuindo fortemente para as mudanças climáticas (gases estufas).

O uso de energias renováveis vem contribuir para redução das emissões dos gases estufa. Uma das fontes de energia com grande potencial no Brasil é a biomassa, a qual se constitui em resíduos agrícolas, tanto animal quanto vegetal, resíduos de agroindústrias, e culturas energéticas (Rêgo et al., 2020; Brito et al., 2020; Queiroz et al., 2021).

Com o aumento pela demanda de alimentos no Mundo, especialmente a proteína animal impulsionada pela China, o Brasil vem destacando-se com um grande exportador e produtor de carnes. Em quantidade de carnes exportadas (bovina, suína e aves), em 2020, o Brasil passou a ocupar o segundo lugar, com 7,4 milhões de toneladas ou 13,4\% do total mundial. O Brasil é o primeiro maior exportador de carne bovina e de frango, e o quarto maior exportador de carne suína. Com relação aos suínos, o Brasil, em 2020, alcançou a terceira posição mundial na produção, com 41 milhões de cabeças, ou seja, 4,4\% do total (ABPA, 2021; ABIEC, 2021).

No processo de criação de suínos, o qual vai desde a matriz para produção de leitões até a terminação, ocorre a disposição de biomassa residual com alta carga orgânica. As propriedades rurais dedicadas a suinocultura despejam os resíduos gerados em lagoas de tratamento, evitando a disposição em corpos de água. Após lagoa, a maioria dos suinocultores utilizam os resíduos para fertirrigação de pastagens ou culturas.

Algumas propriedades rurais com atividade de suinocultura, ao invés de lagoas utilizam biodigestores rurais para tratamento via digestão anaeróbia do resíduo, os quais são basicamente do tipo fluxo contínuo (a pistão), onde o gasômetro é feito de polietileno de alta densidade (PEAD). O produto gerado é o biogás e o biofertilizante ou digestato. O biofertilizante é armazenado numa outra lagoa e por fim utilizado na fertirrigação e o biogás utilizado como biocombustível (Freitas et al., 2019). 
Contudo, devido ao aumento das tarifas de energia, foca-se, cada vez mais, na geração de energia com biogás para o consumo próprio. A maioria das propriedades de suinocultura tem potencial para instalar um motor gerador com potência de até $75 \mathrm{~kW}$, ou seja, na microgeração distribuída. São propriedades com até 6000 suínos alojados.

Em 2012 a ANEEL - Agência Nacional de Energia Elétrica, com o intuito de normatizar a geração distribuída de eletricidade com fontes renováveis, publicou a Resolução Normativa (RN) no 482/2012, que permite a produção de energia elétrica por meio de pequenas centrais geradoras (micro e minigeração de energia), instituiu o Sistema de Compensação de Energia Elétrica. Tal sistema permite que a energia elétrica gerada seja utilizada na própria unidade consumidora e o excedente seja injetado na rede, para que nas próximas faturas seja compensado como crédito de energia (Weirich, 2021).

De acordo com Souza (2016), os motores geradores com potência abaixo de $50 \mathrm{~kW}$ utilizados, são pequenos motores a ignição por centelha adaptados para biogás. Os motores com potências acima de $50 \mathrm{~kW}$, por sua vez, são ciclo diesel (ignição por compressão) convertidos para ignição por centelha à gás (Ciclo Otto), por meio da mudança de taxa de compressão, alteração do ponto e introdução de um sistema de injeção de biogás/ar.

Uma outra opção de geração de eletricidade com biogás, seria o uso de motores geradores ciclo diesel operando no modo dual, o qual caracteriza-se por operar simultaneamente com o combustível líquido (diesel ou biodiesel) e combustíveis gasosos (metano, gás liquefeito de petróleo, hidrogênio e o biogás) (Suzuki et al., 2011; Elnajjar et al., 2013).

Dentre as vantagens do uso dos motores dual diesel/biodiesel e biogás seriam: (Saleh et al., 2008; Barik e Murugan, 2015) a necessidade de poucas adaptações, ou seja, a instalação de um misturador de gás ao ar de admissão; uso do biogás disponível em biodigestores já instalados; o motor operar normalmente apenas com diesel quando não houver biogás; autoprodução de energia elétrica (Saleh et al., 2008; Barik e Murugan, 2015).

No motor gerador tipo dual, ao operar com biogás, o diesel funciona como um combustível piloto. Ocorre uma redução do consumo de diesel entre 10 e 90\%, reduzindo o gasto com diesel mineral ao utilizar o biogás disponível na digestão anaeróbia (Colle, 2018).

Esse trabalho teve como objetivo a análise da viabilidade econômica da geração de energia elétrica, por meio do motor dual, utilizando o biogás e diesel como combustíveis.

\section{Metodologia}

A pesquisa foi desenvolvida na UNIOESTE (Universidade Estadual do Oeste do Paraná), na região oeste do Paraná. O cálculo de viabilidade econômica foi realizado para plantas de produção de eletricidade no modo dual com escalas de 30, 40, 50, 60, 70 e $80 \mathrm{~kW}$. O custo capital compreende desde a instalação do biodigestor até o motor gerador e painéis elétricos. Com dados de custos de fornecedores de motores geradores e biodigestores (Biogas, 2021; BioKholer, 2021), foi elaborada a equação que correlaciona a capacidade da planta $(\mathrm{kW})$ com o custo específico $\left(\mathrm{US} \$ \mathrm{~kW}^{-1}\right)$ :

$$
\mathrm{Ce}=10673 . \text { Pot }^{-0.649}
$$

Onde: Ce é o custo específico da planta $\left(\mathrm{US} \$ . \mathrm{kW}^{-1}\right)$, Pot é a potência da planta $(\mathrm{kW})$.

Verificou-se que o custo específico das plantas de produção de eletricidade decrescem quanto maior a escala da planta, devido a economia de escala.

A partir desses dados, foram calculados os parâmetros de avaliação econômica, tais como o custo de geração de eletricidade, valor presente líquido, tempo de retorno e taxa de retorno, conforme Callaghan (1993), Oliva (2003) e Souza et al. (2013).

Para fins de avaliação econômica, foi calculado o custo de geração de eletricidade com a seguinte equação:

$$
C E=\frac{C C+C M+C O}{E G}
$$


Onde: CC é o custo do capital (R\$/ano); CM é o custo de manutenção (R $\$ / a n o) ; C O$ é o custo de operação (R\$/ano); EG é a energia gerada pelo motor (kWh/ano).

Para o cálculo do custo do capital (CC), R\$/ano, utilizou-se a seguinte equação:

$C C=I_{0} . F R C$

Onde: $\mathrm{I}_{\mathrm{o}}$ consiste no investimento inicial no sistema de geração (US\$); FRC

Para cálculo do investimento inicial de geração $\left(\mathrm{I}_{0}\right)$, utilizou-se a seguinte equação:

$I_{0}=C_{e^{*}}$ Pot

Já o fator de recuperação de capital (FRC), é baseado na série uniforme de pagamentos e é função da taxa de desconto (d) e do número de anos de vida do empreendimento (n).

$$
F_{R}=\left[\frac{d(1+d)^{n}}{(1+d)^{n}-1}\right]
$$

O custo de manutenção (CM), US\$.ano ${ }^{-1}$, correspondeu a uma percentagem do valor de investimento inicial $\left(\mathrm{I}_{0}\right)$ para cada ano de funcionamento do motor, dado em US\$.ano ${ }^{-1}$.

$$
C M=\left(\frac{M}{100}\right) \cdot I_{0}
$$

O custo de operação (CO), US\$.ano ${ }^{-1}$, correspondeu ao produto do consumo de combustível, Cons, (L.ano $\left.{ }^{-1}\right)$ com o $^{2}$ custo do combustível, $\mathrm{C}_{\text {comb }}\left(\mathrm{R} \$ \cdot \mathrm{L}^{-1}\right)$.

$$
\text { CO }=\text { Cons. } C_{\text {comb }}
$$

O custo do combustível ( $\mathrm{C}_{\text {comb }}$ ), US\$/Litro, foi adotado com 0,90 US\$.L-1, o qual seria a média adotada nas bombas de gasolina em Dezembro de 2021.

Para obter o consumo anual de diesel (Cons) utilizou-se a equação:

\section{Cons $=$ Con.t. 3600}

Onde: t é o tempo de operação da planta em h.ano ${ }^{-1}$.

O cálculo do consumo (Con), em L.s ${ }^{-1}$, foi obtido pela seguinte equação:

Con $=\frac{E M}{P C I}$. Dens. 1000

Onde: EM é a energia consumida pelo motor do motor em função de sua eficiência ( $20 \%$ de eficiência), em kJ/s; PCI é o poder calorifico do diesel, $42826 \mathrm{~kJ} / \mathrm{kg}$, Dens é a densidade do Diesel, $850 \mathrm{~m}^{3} \cdot \mathrm{kg}^{-1}$, ANP (2018).

A energia gerada (EG) por cada potência de planta, foi obtida pela equação:

$E G=$ Pot.t

Onde: Pot é a potência da planta, em kW; t é o tempo de operação da planta em h.ano-1.

O tempo de operação da planta t é obtido em função do número de horas diárias de operação da planta:

$t=0 D .365$

Onde: OD é o tempo de operação diária $\left({\mathrm{h} \cdot \mathrm{dia}^{-1}}^{-1}\right)$, onde para este estudo foram adotados 5, 10 e 20 h.ano ${ }^{-1}$. 


\section{Resultados e Discussão}

Basicamente foram encontrados os custos de geração de eletricidade utilizando o motor gerador operando somente cm diesel (diesel 100\%), no modo dual com 50\% de biogás e 50\% de diesel (diesel 50\%) e no modo dual com $90 \%$ de biogás e 10\% de diesel (diesel 10\%) (Colle, 2018).

A Figura 1 e Tabela 1, apresentam o custo da energia gerada para cada potência de motor gerador, quando se utiliza um motor gerador operando no modo dual consumindo $100 \%$ de diesel e sem consumo de biogás, nas situações: operação da planta de 5 horas por dia $\left(5\right.$ h.dia $\left.{ }^{-1}\right) ; 10$ horas por dia $\left(10\right.$ h.dia $\left.{ }^{-1}\right) ; 20$ horas por dia $\left(20\right.$ h.dia $\left.{ }^{-1}\right)$. Os resultados são mostrados em dólar americano (US\$). A tarifa de consumo da COPEL (Companhia Paranaense de Energia Elétrica) modalidade rural (B1) é $\mathrm{R} \$ 537,90 . \mathrm{MWh}^{-1}$, ou US\$ 96,00. $\mathrm{MWh}^{-1}$, com o 1 US\$ a R \$ 5,60. Observa-se que quando utiliza-se Diesel puro, a um valor de US\$ $0,90 \mathrm{~L}^{-1}$ ou R $\$ 5,00 . \mathrm{L}^{-1}$, não há viabilidade pois o custo mínimo de geração é de US\$510,77 $\mathrm{kWh}^{-1} \mathrm{com}$ a planta operando numa potência de $80 \mathrm{~kW}$ e 20 horas de funcionamento, ou seja, cinco vezes maior que a tarifa de energia da concessionária.

Figura 1. Custo da energia gerada para cada potência de geração, quando se utiliza um motor gerador operando no modo dual consumindo $100 \%$ de diesel.

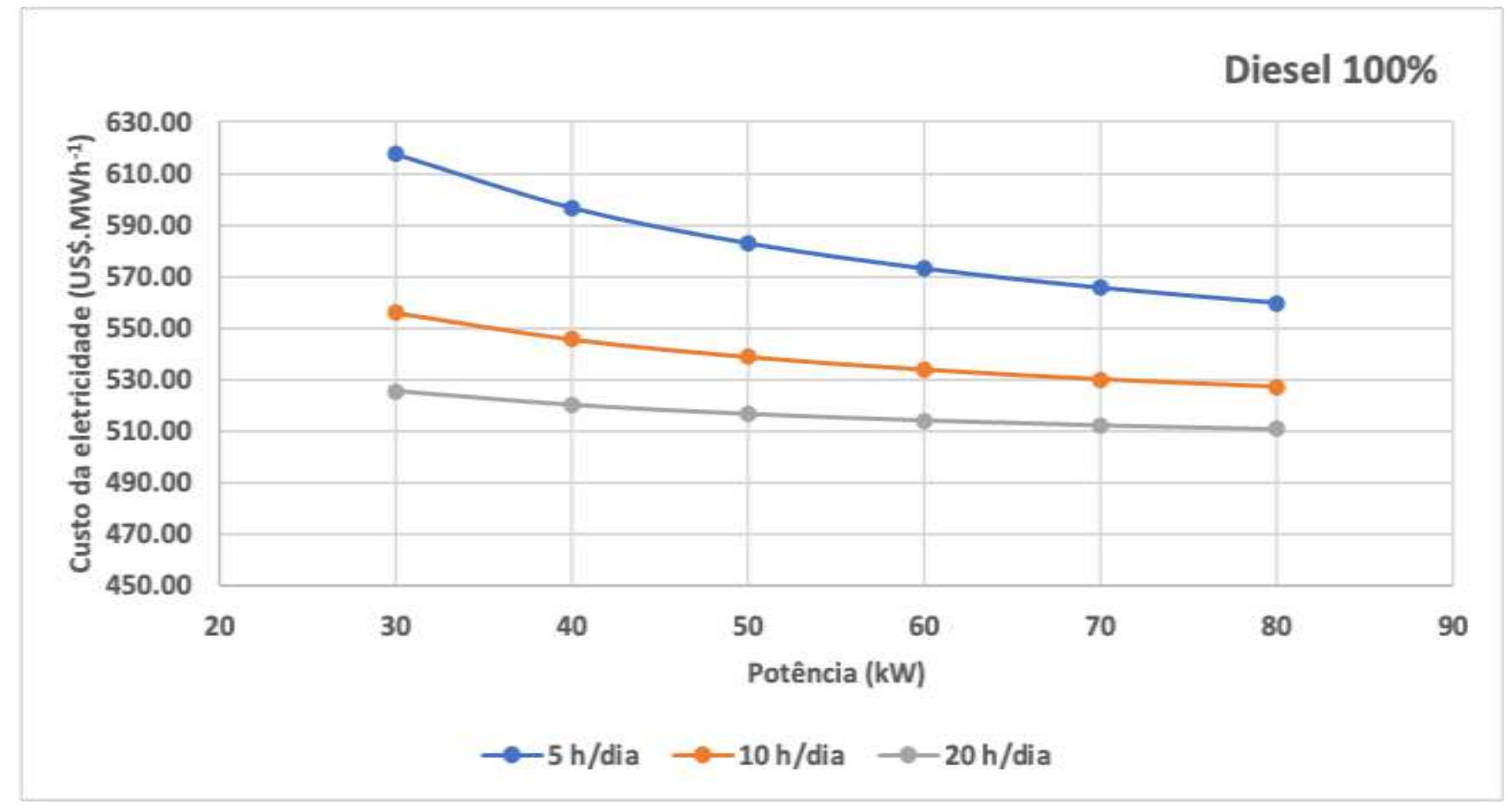

Fonte: Autores. 
Tabela 1. Valores do custo de geração de eletricidade utilizando motor dual consumindo $100 \%$ de diesel e sem consumo de biogás.

\begin{tabular}{cccc}
\hline & \multicolumn{3}{c}{ Custo de geração de eletricidade (US\$ MWh $\left.\mathbf{~}^{-1}\right)$} \\
\hline Potência $(\mathbf{k W})$ & $\mathbf{5 h} / \mathbf{d i a}$ & $\mathbf{1 0 h} / \mathbf{d i a}$ & $\mathbf{2 0 h} / \mathbf{d i a}$ \\
30 & 617,64 & 556,06 & 525,26 \\
40 & 596,66 & 545,57 & 520,02 \\
50 & 582,89 & 538,68 & 516,58 \\
60 & 573,02 & 533,75 & 514,11 \\
70 & 565,54 & 530,00 & 512,24 \\
80 & 559,64 & 527,06 & 510,77 \\
\hline
\end{tabular}

Fonte: Autores.

A Figura 2 e Tabela 2, apresentam o custo da energia gerada com o motor gerador operando no modo dual consumindo $50 \%$ de diesel e $50 \%$ de biogás, operando 5 horas por dia $\left(5\right.$ h.dia $\left.{ }^{-1}\right), 10$ horas por dia $\left(10\right.$ h.dia $\left.{ }^{-1}\right)$ e 20 horas por dia (20 h.dia $\left.{ }^{-1}\right)$ para diferentes capacidades de produção em kW. A tarifa de consumo da COPEL modalidade rural (B1) é R\$ 537,90. $\mathrm{MWh}^{-1}$, ou US\$ 96,00. $\mathrm{MWh}^{-1}$, com o 1 US\$ a R\$ 5,60. Portanto não é viável que o motor opere no modo dual com consumo de $50 \%$ de diesel, pois os custos de produção em todas as situações são maiores que a tarifa para no meio rural. $\mathrm{O}$ alto preço do diesel inviabiliza a geração.

Figura 2. Custo da energia gerada para cada potência de geração, quando utiliza-se um motor gerador operando no modo dual consumindo $50 \%$ de diesel e $50 \%$ de biogás.

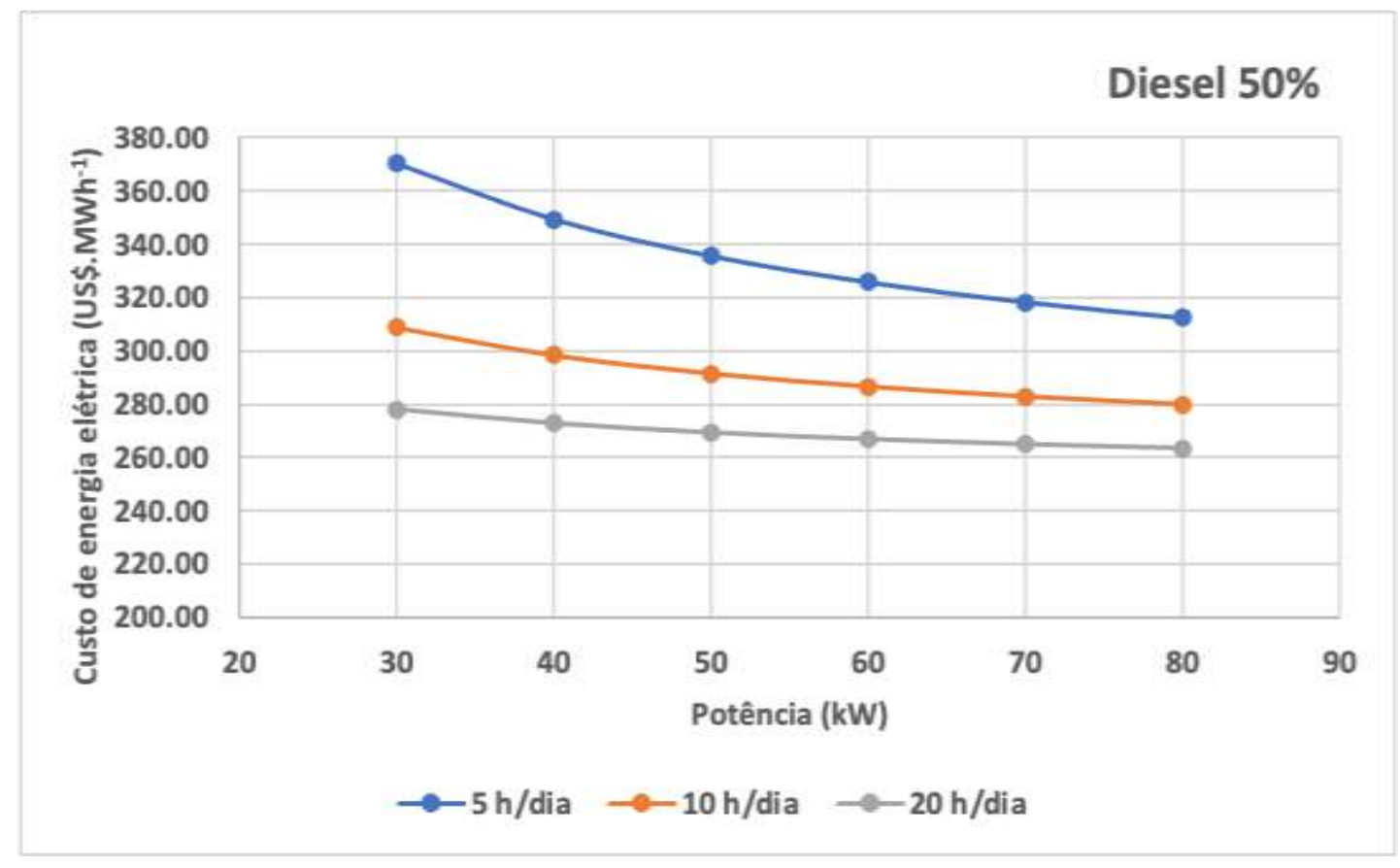

Fonte: Autores. 
Tabela 2. Valores do custo de geração de eletricidade utilizando motor dual consumindo $50 \%$ de diesel e $50 \%$ biogás.

\begin{tabular}{|c|c|c|c|}
\hline \multirow[b]{2}{*}{ Potência $(\mathbf{k W})$} & \multicolumn{3}{|c|}{ Custo de geração de eletricidade (US\$ MWh'-1) } \\
\hline & $5 \mathrm{~h} / \mathrm{dia}$ & $10 \mathrm{~h} / \mathrm{dia}$ & $20 \mathrm{~h} / \mathrm{dia}$ \\
\hline 30 & 370,40 & 308,82 & 278,03 \\
\hline 40 & 349,42 & 298,33 & 272,78 \\
\hline 50 & 335,65 & 291,44 & 269,34 \\
\hline 60 & 325,78 & 286,51 & 266,87 \\
\hline 70 & 318,30 & 282,77 & 265,00 \\
\hline 80 & 312,40 & 279,82 & 263,53 \\
\hline
\end{tabular}

Fonte: Autores.

A Figura 3 e Tabela 3, apresentam o custo da energia gerada para diferentes capacidades de geração, quando utiliza-se um motor gerador operando no modo dual consumindo 10\% de diesel e 90\% biogás. Ou seja, a dependência do diesel diminui, viabilizando a geração nas situações onde a planta ter potência de 50, 60, 70 e $80 \mathrm{~kW}$ e operar 10 horas por dia ou, a planta operar 20 horas por dia em plantas com potência entre 30 e $80 \mathrm{~kW}$.

Figura 3. Custo da energia gerada para cada potência de geração, quando se utiliza um motor gerador operando no modo dual consumindo $10 \%$ de diesel e $90 \%$ de biogás.

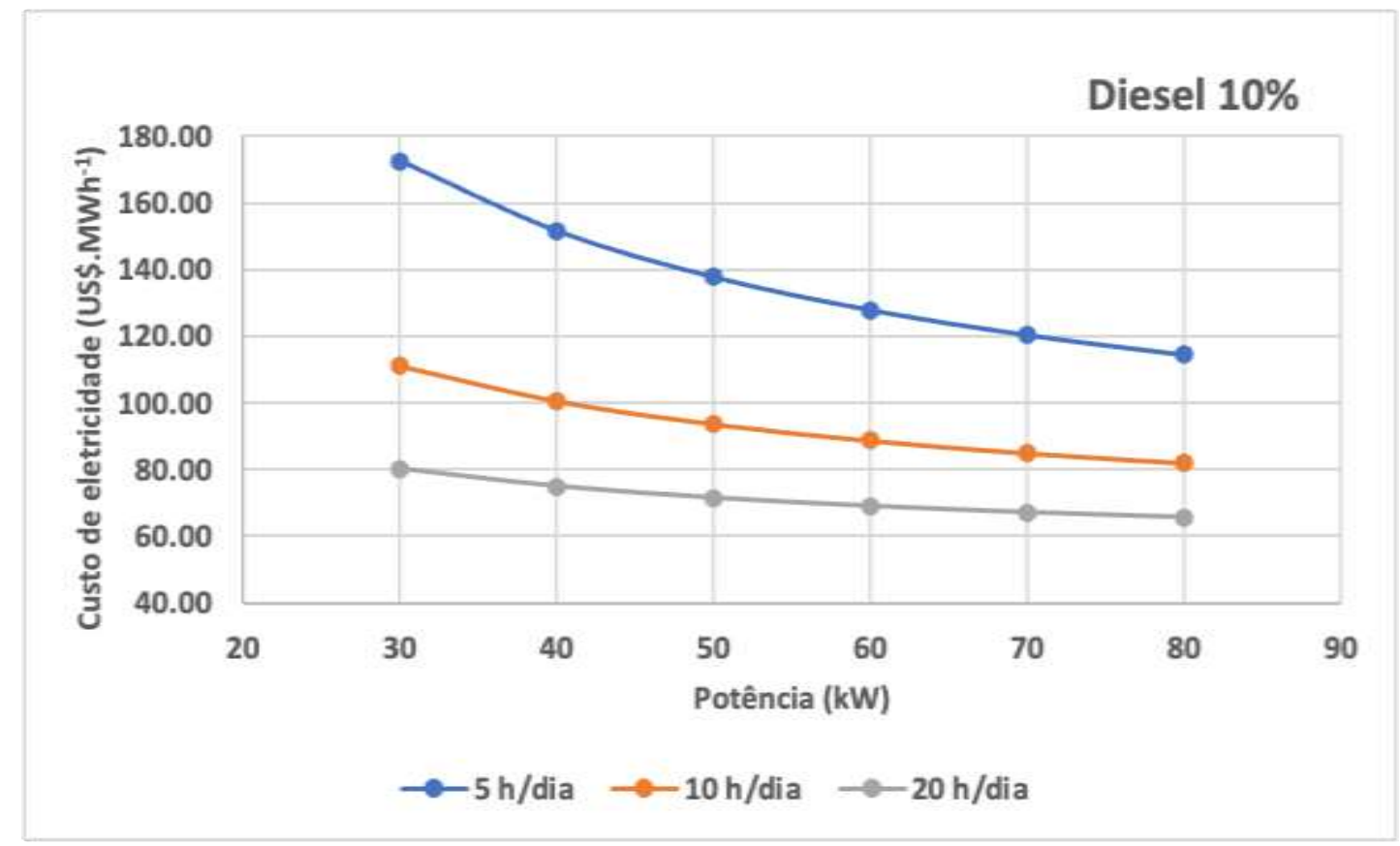

Fonte: Autores. 
Tabela 3. Valores do custo de geração de eletricidade utilizando motor dual consumindo $10 \%$ de diesel e $90 \%$ biogás.

\begin{tabular}{|c|c|c|c|}
\hline \multirow[b]{2}{*}{ Potência (kW) } & \multicolumn{3}{|c|}{ Custo de geração de eletricidade (US\$ $M W^{-1}$ ) } \\
\hline & $5 \mathrm{~h} / \mathrm{dia}$ & 10h/dia & $20 \mathrm{~h} / \mathrm{dia}$ \\
\hline 30 & 172,61 & 111,03 & 80,23 \\
\hline 40 & 151,63 & 100,54 & 74,99 \\
\hline 50 & 137,85 & 93,65 & 71,55 \\
\hline 60 & 127,99 & 88,72 & 69,08 \\
\hline 70 & 120,51 & 84,98 & 67,21 \\
\hline 80 & 114,62 & 82,03 & 65,74 \\
\hline
\end{tabular}

Fonte: Autores.

\section{Conclusão}

O custo de geração de eletricidade diminui com a escala da planta de geração, em kW. Maiores escalas menor o custo.

Quando dependência do diesel diminui, ou seja, para plantas no modo dual ajustadas para consumir $90 \%$ de biogás e somente $10 \%$ de diesel, verificou-se que nas situações onde a planta tem potência acima de 50 e operaram 10 horas por dia há viabilidade de geração de eletricidade, onde os custos são competitivos com a tarifa praticada pela concessionária.

\section{Referências}

ABIEC - Associação Brasileira das Indústrias Exportadoras de Carnes. Beef Report-Perfil da pecuária no Brasil 2021. 60p. http://abiec.com.br/publicacoes/beef-report-2021.

ABPA - Associação Brasileira de Proteína Animal. Relatório Anual (2021), 146p. http://abpa-br.org/mercados/\#relatorios.

Agência Nacional do Petróleo, Gás Natural e Biocombustíveis - ANP (2018). Sistema de levantamento de preços. http://anp.gov.br/preco/prc/Resumo_Por_Estado_Municipio.asp.

Barik, D., Murugan, S. (2015). Experimental investigation on the behavior of a DI diesel engine fueled with raw biogas-diesel dual fuel at different injection timing. Journal of the Energy Institute. 89, 373-388.

Biogás. Biogás Motores Estacionários. Consulta sobre orçamentos de plantas a biogás. 2021.

BioKholer. Consulta sobre orçamentos de plantas a biogás. 2021.

Brito, M. R., Outa, R., Chavarette, F. R., Gonçalves, A. C., de Albuquerque, M. D. C. F., \& de Souza Miranda, R. (2020). Análise das propriedades do biodiesel da polpa do abacate como lubrificante: teste pin on disk. Research, Society and Development, 9(7), e136973886-e136973886.

Callaghan, P. O. (1993). Energy Management. McGraw-Hill. London.

Colle, D. Avaliação de desempenho energético de um gerador elétrico operando no modo dual, utilizando biogás e blendas de biodiesel de óleo residual de fritura. 2018. Dissertação (Mestrado em Engenharia de Energia na agricultura) - Universidade Estadual do Oeste do Paraná.

Colle, Daniel et al. Avaliação de desempenho energético de um gerador elétrico operando no modo dual, utilizando biogás e blendas de biodiesel de óleo residual de fritura. 2018.

Elnajjar, E., Selim, M. Y., \& Hamdan, M. O. (2013). Experimental study of dual fuel engine performance using variable LPG composition and engine parameters. Energy conversion and Management, 76, 32-42.

Freitas, F. F., De Souza, S. S., Ferreira, L. R. A., Otto, R. B., Alessio, F. J., De Souza, S. N. M., ... \& Junior, O. A. (2019). The Brazilian market of distributed biogas generation: Overview, technological development and case study. Renewable and Sustainable Energy Reviews, 101, 146-157.

Queiroz, I. R., da Costa, A. S. V., Almeida, I. C., Barros, G. F., Alves, W. M., de Souza, M. C., ... \& Ferreira, A. C. (2021). O biocombustível no Brasil: potencialidades da cultura do Crambe abyssinica para produção de biodiesel. Research, Society and Development, 10(5), e11510514618-e11510514618.

Oliva, C. A. (2003). Geração própria de energia elétrica com aproveitamento de biogás derivado da suinocultura. Dissertação de Mestrado, Programa de Pós-Graduação em Engenharia Agrícola. Universidade Estadual do Oeste do Paraná.

Rêgo, D. M. G., da Silva, L. C., de Morais Oliveira, M. N., de Melo, R. P. F., \& Carmo, S. K. S. (2020). Planejamento fatorial e avaliação econômica do processo de produção de biodiesel a partir da gordura suína. Research, Society and Development, 9(9), e321997263-e321997263. 
Research, Society and Development, v. 10, n. 17, e106101724528, 2021

(CC BY 4.0) | ISSN 2525-3409 | DOI: http://dx.doi.org/10.33448/rsd-v10i17.24528

Saleh H. E. (2008). Effect of variation in LPG composition on emissions and performance in a dual fuel diesel engine. Fuel. 87, $3031-3019$.

Silva, F. P. Universidade Estadual do Oeste do Paraná, Março de 2015. Eficiência energética de uma unidade de microgeração de energia elétrica a partir do biogás da suinocultura.

Souza, S. N. M. (2016). Manual de geração de energia elétrica a partir do Biogás no meio rural. Dissertação de Mestrado, Programa de Pós-Graduação em Engenharia de Energia na Agricultura. Universidade Estadual do Oeste do Paraná.

Souza, S. N. M., Werncke, I., Marques, C. A., Bariccatti, R. A., Santos, R. F., Nogueira, C. E. C., \& Bassegio, D. (2013). Electric energy micro-production in a rural property using biogas as primary source. Renewable and Sustainable Energy Reviews, 28, 385-391.

Suzuki P. B. A., Fernandes M. D., Faria P. A. R, Morais Vidal M. C. T. (2011). Uso de biogás em motores de combustão interna. Revista Brasileira de Tecnologia Aplicada nas Ciências Agrárias. 4, 221-237.

Weirich, C. S. Análise econômica de sistemas de geração de eletricidade no modo GD: motor gerador a biogás e painéis fotovoltaicos. 2021. Dissertação (Mestrado em Engenharia de Energia na Agricultura - PPGEA) - Universidade Estadual do Oeste do Paraná. 